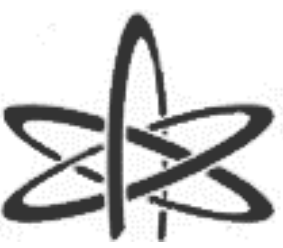

BJRS
BRAZILIAN JOURNAL

$\mathrm{OF}$

RADIATION SCIENCES

07-2A (2019) 01-09

\title{
Feasibility studies of using thin entrance window photodiodes for clinical electron beam dosimetry
}

\author{
C. R. Nascimento ${ }^{\mathrm{a}, \mathrm{b}}$; V. K. Asfora ${ }^{\mathrm{a}}$; V. S. M. Barros ${ }^{\mathrm{a}}$; J. A. C. Gonçalves ${ }^{\mathrm{c}}$; \\ L. F. R. Andrade ${ }^{\text {d }}$ H. J. Khoury ${ }^{\mathrm{a}}$; C. C. Bueno ${ }^{\mathrm{c}}$ \\ ${ }^{a}$ Departamento de Energia Nuclear - Universidade Federal de Pernambuco (UFPE) \\ 50740-540 Recife-PE, Brazil \\ ${ }^{b}$ Instituto Federal de Educação, Ciência e Tecnologia de Pernambuco-IFPE, Recife-PE, Brazil \\ ${ }^{c}$ Instituto de Pesquisas Energéticas e Nucleares (IPEN / CNEN - SP) \\ 05508-000 São Paulo, SP, Brazil \\ ccbueno@ipen.br \\ ${ }^{d}$ Santa Casa de Misericórdia de Itabuna. Rua Santa Cruz, s/n, 45603-305, Fátima, Itabuna-BA, Brazil
}

\begin{abstract}
The response of the commercial XRA-24 PIN photodiode $\left(5.76 \mathrm{~mm}^{2}\right.$ active area) for clinical electron beam dosimetry covering the range of 8-12 MeV was investigated. Within this energy range, the charge generated in the diode's sensitive volume is linearly dependent on the absorbed dose up to 320 cGy. However, charge sensitivity coefficients evidenced that the dose response of the diode is slightly dependent on the electron beam energy. Indeed, the diode's energy dependence was within $8.5 \%$ for 8-12 MeV electron beams. On the other hand, it was also observed an excellent repeatability of these results with a variation coefficient (VC) lower than 0.4\%, which is within the $1 \%$ tolerance limit recommended by the AAPM TG-62. Furthermore, the agreement between the percentage depth dose profiles (PDD) gathered with the diode and the ionization chamber allowed achieving the electron beam quality within $1 \%$ of that obtained with the ionization chamber. Based on these results, the photodiode XRA-24 can be a reliable and inexpensive alternative for electron beams dosimetry.
\end{abstract}

Keywords: Electron dosimetry, Si photodiodes dosimeters, Solid-state dosimetry. 


\section{INTRODUCTION}

Silicon diodes have been widely employed in clinical photon beam dosimetry owe to a number of advantages over air-filled ionization chambers, commonly used for absolute and relative dose measurements. Among these advantages prevails the high sensitivity to ionizing radiation per unit volume of silicon which allows for design of compact-sized diodes. Such diodes are available with active area less than a few square millimeters and therefore with proper spatial resolution to be used in small field photons dosimetry [1-4]. Moreover, the progress in device design and fabrication technology has widened the range of applications of silicon diodes especially in those which require rather thin dead layer, such as, for example, soft X-ray detection and dosimetry [5-6].

In this context, several manufacturers have been involved in developing thin entrance window devices and one type of them, the PIN photodiode XRA-24 (Detection Technology Inc.) has proven to attain good performance in clinical photon beam dosimetry with almost negligible energy dependence [7]. Taking into account this result and the features of these diodes, it seems quite feasible to employ them in electron dosimetry. In this context, the aim of this work was to study the XRA-24 diode's response for clinical electron beam dosimetry by investigating the following main dosimetry parameters: repeatability, charge versus dose response, energy dependence and percentage depth dose profile (PDD).

\section{EXPERIMENTAL SETUP}

In this work, the photodiode XRA-24, with active area of $5.76 \mathrm{~mm}^{2}(2.4 \mathrm{~mm} \times 2.4 \mathrm{~mm})$ was housed in a homemade polymer plastic probe covered by a $100 \mathrm{mg} / \mathrm{cm}^{2}$ black plastic layer. This probe was inserted in a depression at the center of a $30 \mathrm{~cm}$ x $30 \mathrm{~cm}$ polymethyl methacrylate (PMMA) plate with $1 \mathrm{~cm}$ thickness, providing the diode's front side layer leveled with the surface of the plate. To perform the measurements, the $\mathrm{n}^{+}$backside of the diode was kept grounded while the $\mathrm{p}^{+}$front pad was connected in a short circuit mode to the input of an integrating electrometer (Standard Imaging model CDX 2000A).

The irradiations were performed with 8, 10 and $12 \mathrm{MeV}$ electron beams from a Siemens Energy Mid Primus linear accelerator coupled to an electron cone applicator of $10 \mathrm{~cm}$ x $10 \mathrm{~cm}$. Unless otherwise stated, during all measurements the diode was held between PMMA plates, placed at the reference depth and centered in a radiation field of $10 \mathrm{~cm}$ x $10 \mathrm{~cm}$, with the source-to-surface 
distance (SSD) kept at $100 \mathrm{~cm}$. For each of electron beam energies, the plastic reference depth was obtained from the water reference depth in accordance with IAEA/TRS-398 recommendations [8]. Firstly, the response repeatability of the diode was investigated for all electron beam energies. For each one, ten measurements were consecutively registered for the same radiation dose of $100 \mathrm{cGy}$, just switching on and off the electron beam. The repeatability performance was analyzed based on the variation coefficients (VC), obtained by the ratio between the standard deviation (SD) and the average reading.

To achieve the dose-response curve of the XRA-24 diode, given by the charge as a function of the absorbed dose, five consecutive measurements were carried out for each monitor unit value covering the range of 5 to $320 \mathrm{cGy}$. The absolute dose measurements were carried out using a calibrated parallel plate ionization chamber (Markus PTW 30013) coupled to an electrometer. The absorbed dose to water was calculated according to the International Atomic Energy Agency's absolute dosimetry protocol (Technical Reports Series 381), by applying appropriate correction factors for a beam quality and environment [9].

The dose-response curves were gathered for 8, 10 and $12 \mathrm{MeV}$ electron beams and apart from the linearity analysis, the sensitivity coefficients ( $\mathrm{nC} / \mathrm{cGy}$ ) of the diode were obtained for each of electron beam energies. The diode's sensitivity dependence on the electron beam energy was also studied.

The percentage of depth dose (PDD) profiles of 8, 10 and $12 \mathrm{MeV}$ electron beams were carried out in a PMMA phantom by changing the depth of the diode from 0 (phantom surface) to $50 \mathrm{~mm}$ with PMMA plates, with SSD kept at $100 \mathrm{~cm}$. For each position, two measurements were performed with a constant dose of $100 \mathrm{cGy}$. To obtain the PDD profiles, the dose measured at each depth was divided by that measured at the maximum dose depth and multiplied by 100 . The experimental results gathered with the photodiode were compared with those obtained with the ionization chamber (Markus PTW 30013), measured in the water phantom. The PMMA thickness was converted to water thickness following IAEA/TRS-398 [8] recommendations. To give more substance to this comparison, values of mean energy of the electrons at the phantom surface $\left(\mathrm{E}_{0}\right)$, the practical range $\left(R_{p}\right)$ and the half-value depth $\left(R_{50}\right)$ were obtained from the PDD curves of the diode and ionization chamber as well.

In all measurements, the combined uncertainties of the results were calculated with the well-known error propagation formula taking into account the diode's reading uncertainty, the accelerator's stability, both resolution and stability of the electrometer. 


\section{RESULTS AND DISCUSSIONS}

\subsection{Repeatability}

The average of ten consecutive measurements gathered at $100 \mathrm{cGy}$ for 8,10 and $12 \mathrm{MeV}$ electron beams, with both correspondent standard deviation (SD) and variation coefficient (VC) are shown in Table 1. As can be seen, the VC values lower than $0.2 \%$, fulfil the requirements of the AAPM protocol TG-62 [10], which recommends repeatability better than $1 \%$ for diodes used as dosimeters. These results are similar to those $(\mathrm{VC}<0.5 \%)$ obtained with the diode of Scanditronix and reported by Soriani et al. [11]. The response repeatability of the photodiode XRA-24 is better than that obtained with the photodiode BPW-34 employed in electron dosimetry [12].

Table 1: Mean reading of the diode, at $100 \mathrm{cGy}$, with the standard deviation (SD) and variation coefficient (VC) for 8,10 and $12 \mathrm{MeV}$ electron beams.

\begin{tabular}{cccc}
\hline $\begin{array}{c}\text { Energy } \\
(\mathrm{MeV})\end{array}$ & $\begin{array}{c}\bar{M}_{\text {Diode }} \\
(\mathrm{nC})\end{array}$ & $\begin{array}{c}\mathrm{SD} \\
(\mathrm{nC})\end{array}$ & $\begin{array}{c}\mathrm{VC} \\
(\%)\end{array}$ \\
\hline 8 & 1565.34 & 1.80 & 0.11 \\
10 & 1454.00 & 2.13 & 0.15 \\
12 & 1438.90 & 2.02 & 0.14 \\
\hline
\end{tabular}

\subsection{Dose-Response and Energy Dependence}

Figure 1 shows the charge generated in the diode as a function of the absorbed doses covering the range of 5-320 cGy. Each point corresponds to the mean value of five consecutive measurements carried out with the photodiode. The combined uncertainties associated with each value are lower than $0.8 \%$ for $\mathrm{k}=2$.

As shown in Figure 1, the dose-response curves for all electron beam energies are quite linear (data fitting $\mathrm{R}^{2}$ coefficient higher than 0.99958 ) with charge sensitivity coefficients (nC/cGy), obtained from the angular coefficient of each dose-response curve, presented in Table 2.

Figure 1: Dose-response curves of the diode for 8, 10 and $12 \mathrm{MeV}$ electron beam energy. 


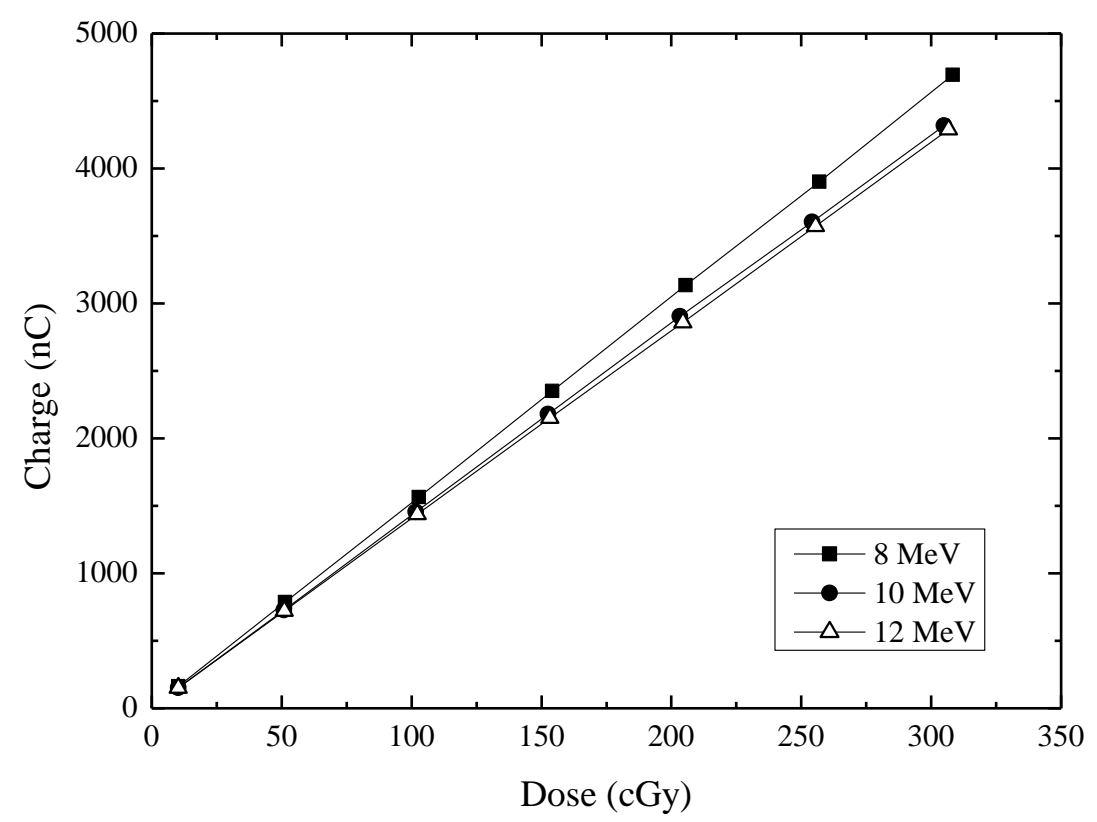

Table 2: Charge Sensitivity coefficients of the XRA-24 photodiode for 8, 10 and $12 \mathrm{MeV}$ electron beam.

\begin{tabular}{cc}
\hline $\begin{array}{c}\text { Energy } \\
(\mathrm{MeV})\end{array}$ & $\begin{array}{c}\text { Sensitivity Coefficient } \\
(\mathrm{nC} / \mathrm{cGy})\end{array}$ \\
\hline 8 & $15.33 \pm 0.04$ \\
10 & $14.34 \pm 0.03$ \\
12 & $14.13 \pm 0.03$ \\
\hline
\end{tabular}

The variation of these sensitivity coefficients shows the dependence of the XRA-24 response on the electron beam energy. It is evidenced in Figure 2, where the charge sensitivity of $8 \mathrm{MeV}$ electron beam is almost $8.5 \%$ higher than that obtained for $12 \mathrm{MeV}$ electron beam. This energy dependence might be due to the difference of the deposited energy in the diode's sensitive volume by 8 and 12 $\mathrm{MeV}$ electron beams.

\subsection{Percentage depth dose (PDD) profile}

Figure 3 shows the percentage depth dose (PDD) curves carried out with the photodiode XRA-24 together with those obtained with the ionization chamber for 8,10 and $12 \mathrm{MeV}$ electron beam energies. For each of electron energies, the comparison of both dosimeters shows good agreement 
for PDD values higher than 25\%. However, in the lower PDD region the response of the photodiode is $10 \%$ lower than that obtained with the ionization chamber.

The mean energy of the electrons at the phantom surface $\left(E_{0}\right)$, the practical range $\left(R_{p}\right)$ and the halfvalue depth $\left(\mathrm{R}_{50}\right)$ were also estimated from Figure 3. The value of $E_{0}$ was obtained through the relationship obtained by the IAEA/TRS 381 [9]:

$$
\mathrm{E}_{0}=0.656+2.059 \mathrm{R}_{50}+0.022\left(\mathrm{R}_{50}\right)^{2}
$$

Figure 2: Charge sensitivity coefficients of XRA-24 diode as a function of electron beam energies.

The spline line is just a guide to the eyes.

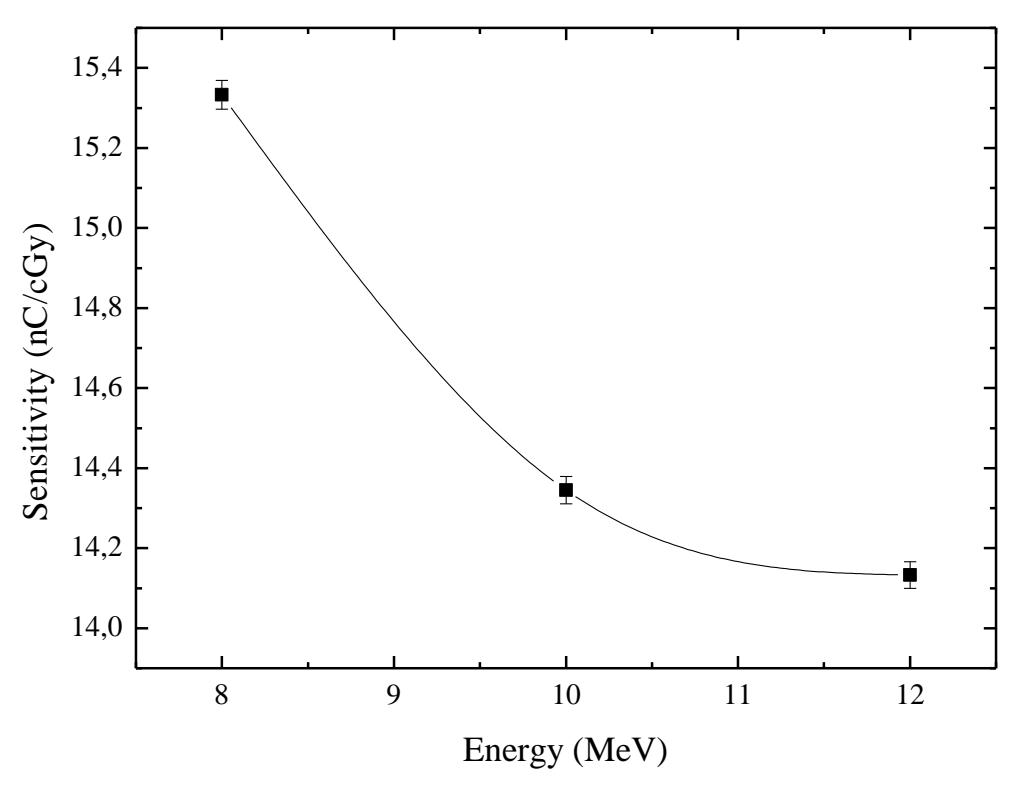

As can be seen in Table 3, all the results obtained with the diode and the ionization chamber agreed within $2 \%$ which is comparable with the uncertainty values.

Figure 3: Comparison between percentage depth dose (PDD) in water for 8, 10 and $12 \mathrm{MeV}$ electron beam measured with XR-24 and ionization chamber Markus PTW 30013. 


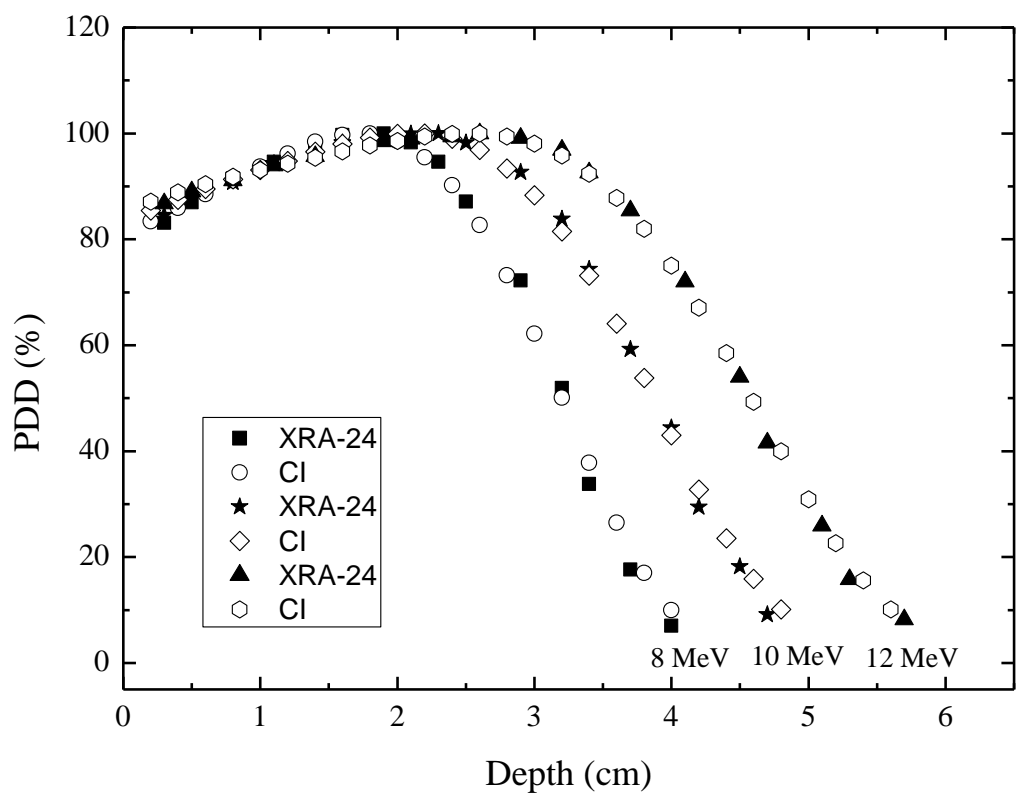

Table 3: Mean energy of the electrons at the phantom surface $\left(E_{0}\right)$, the practical range $(R p)$ and the half-value depth (R50) obtained with the XRA 24 and ionization chamber for electron beams of 8,10 and $12 \mathrm{MeV}$.

\begin{tabular}{|c|c|c|c|c|c|c|}
\hline \multirow{2}{*}{$\begin{array}{l}\text { Nominal } \\
\text { Beam } \\
\text { Energy } \\
\text { E }(\mathrm{MeV})\end{array}$} & \multicolumn{3}{|c|}{ Photodiode -XRA-24 } & \multicolumn{3}{|c|}{$\begin{array}{l}\text { Ionization Chamber } \\
\text { Markus PTW } 30013\end{array}$} \\
\hline & $\begin{array}{c}\bar{E}_{0} \\
(\mathrm{MeV})\end{array}$ & $\begin{array}{l}\mathrm{R}_{50} \\
(\mathrm{~cm})\end{array}$ & $\begin{array}{c}\mathrm{R}_{\mathrm{p}} \\
(\mathrm{cm})\end{array}$ & $\begin{array}{c}\bar{E}_{0} \\
(\mathrm{MeV})\end{array}$ & $\begin{array}{c}\mathrm{R}_{50} \\
(\mathrm{~cm})\end{array}$ & $\begin{array}{c}\mathrm{R}_{\mathrm{p}} \\
(\mathrm{cm})\end{array}$ \\
\hline 8 & 7.47 & 3.2 & 4.0 & 7.47 & 3.2 & 4.1 \\
\hline 10 & 8.91 & 3.85 & 4.81 & 8.95 & 3.87 & 4.89 \\
\hline 12 & 10.41 & 4.52 & 5.62 & 10.57 & 4.59 & 5.72 \\
\hline
\end{tabular}

\section{CONCLUSION}

The response of a PIN photodiode XRA-24 for clinical electron beam dosimetry was investigated in the energy range of 8-12 MeV. The results obtained with the diode operating in a photovoltaic mode, evidenced that the charge generated in its sensitive volume increases linearly with the absorbed dose up to $320 \mathrm{cGy}$. It was also observed an excellent repeatability of these results with a 
variation coefficient (VC) lower than $0.4 \%$ and, therefore, within AAPM TG-62 Report 87 recommendations.

Despite of these good results, charge sensitivity coefficients evidenced that the dose response of the diode is slightly dependent on the electron beam energy. Indeed, the diode's energy dependence was within $8.5 \%$ for $8-12 \mathrm{MeV}$ electron beams. Furthermore, the agreement between the percentage depth dose profiles (PDD) gathered with the diode and the ionization chamber allowed achieving the electron beam quality within $1 \%$ of that obtained with the ionization chamber.

Based on these results, the photodiode XRA-24 can be a reliable and inexpensive alternative for electron beams dosimetry. Furthermore, it seems that this diode might be used for in vivo dosimetry as well as in small radiation field applications.

\section{ACKNOWLEDGMENT}

The authors would like to acknowledge the financial support received from the Brazilian National Research Council (CNPq) and the Santa Casa de Misericórdia de Itabuna-Bahia.

\section{REFERENCES}

1. GIESSBACH, I.; LAPP, M.; BOHSUNG, J.; GADEMANN, G.; HARDE, D. Dosimetric characteristics of a new unshield silicon diode and its application in clinical photon and electron beams. Medical Physics, v. 32, p. 12, 2005.

2. ROSENFELD, A. B.; CUTAJAR, D.; LERCH, M. L. F.; TAKACS, G.; CORNELIUS, I. M. ; YUDELEV, M.; ZAIDER, M. Miniature semiconductor detectors for in vivo dosimetry. Radiation Protection Dosimetry, v. 120 (1-4), p. 48-55, 2006.

3. SONG, H.; AHMAD, M.; DENG, J.; CHEN, Z.; YUE, N. J.; NATH, R. Limitations of silicon diodes for clinical electron dosimetry. Radiation Protection Dosimetry, v. 120 (1-4), p. 5659, 2006.

4. SANTOS, T. C.; NEVES, W. F. P.; GONÇAlVES, J. A. C.; HADDAD, C. M. K.; HARKONEN, J.; BUENO, C. C. Characterization of miniature RAD-HARD silicon diodes as dosimeters for small fields of photon beams used in radiotherapy. Radiation Measurements, v. 71, p. 396-401, 2014. 
5. GONÇALVES, J. A. C.; PEREIRA, L. N.; POTIENS, M. P. A.; VIVOLO, V.; BUENO, C. C. Evaluation of epitaxial silicon diodes as dosimeters in X-ray mammography. Radiation Measurements, v. 71, p. 384-388, 2014.

6. BRUZZI, M.; BUCCIOLINI, M.; CASATI, M.; MENICHELlI, D.; TALAMONTI, C.; PIEMEONTE, C.; SVENSSON, B. G. Epitaxial silicon devices for dosimetry applications. Applied Physics Letters, v. 90, p. 172109, 2007.

7. BATISTA, E.; KHOURY, H. J.; MELO, F. A.; BARROS, V.; SIlVA Jr, E. F. The Performance of Commercial Photodiodoes for Dosimetry in Mammography. Radiation Protection Dosimetry, v. 115 (1-4), p. 391-393, 2005.

8. IAEA. Absorbed Dose Determination in External Beam Radiotherapy: An International Code of Practice for Dosimetry Based on Standards of Absorbed Dose to Water. International Atomic Energy Agency Technical Reports Series (IAEA-TRS) n. 398. Vienna, 2000.

9. IAEA. The Use of Plane-Parallel Ionization Chambers in High-Energy and Photons Beams: An International Code of Practice for Dosimetry. International Atomic Energy Agency Technical Reports Series (IAEA-TRS) n. 381. Vienna, 1997.

10. AAPM. Diode in vivo dosimetry for patients receiving external beam radiation therapy. Report of American Association of Physicists in Medicine Radiation Therapy Committee Task Group 62. AAPM- TG-62 Report 87. New York: AAPM, 2005.

11. SORIANI, A.; DE ANGELIS, C.; ONORI, S.; IACCARINO, G.; MARZI, S.; LANDONI, V.; BENASSI, M. Sistemi a stato sólido per la dosimetria di fasci di elettroni ad alto rateo di dose. In: III Congresso Nazionale dell'Associazione italiana di Fisica in Medicina (AIFM), Agrigento, 2003.

12. KHOURY, H. J.; HAZIN, C. A.; MASCARENHAS, A. P.; SILVA Jr, E. F. Low cost silicon photodiode for electron dosimetry. Radiation Protection Dosimetry, v. 84 (1-4), p. 341-343, 1999. 